\title{
A GREEN'S FUNCTION NUMERICAL METHOD FOR SOLVING PARABOLIC PARTIAL DIFFERENTIAL EQUATIONS
}

\author{
LUKE EDWARDS \\ Research Supervisors: Anna L. Mazzucato and Victor Nistor \\ Department of Mathematics, Penn State University, \\ University Park, PA, 16802.
}

\begin{abstract}
This article describes the derivation and implementation of a numerical method to solve constant-coefficient, parabolic partial differential equations in two space dimensions on rectangular domains. The method is based on a formula for the Green's function for the problem obtained via reflections at the boundary of the domain from the corresponding formula for the fundamental solution in the whole plane. It is inspired by a related method for variable coefficients equations in the whole space introduced by Constantinescu, Costanzino, Mazzucato, and Nistor in J. Math. Phys, 51103502 (2010). The benchmark case of the two-dimensional heat equation is considered. We compare the Green's function method with a finite-difference scheme, more precisely, an alternating direction implicit (ADI) method due to Peaceman and Rachford. Our method yields better rates of convergence to the exact solution.
\end{abstract}

1. Introduction 27

2. Fundamental Solution Approach 29

3. Numerical Method 34

4. Comparison Methods 35

5. Results and Conclusions $\quad 37$

$\begin{array}{ll}\text { References } & 39\end{array}$

Contents

\section{INTRODUCTION}

This research is motivated by the desire to maximize calculation speed in engineering, scientific, financial and other applications that necessitate a large amount of approximations in a short amount of time. These applications require numerical methods for approximating solutions to partial differential equations (PDEs) with high accuracy and efficiency.

In this paper, we demonstrate the robustness of a numerical method for solving parabolic PDEs in two space dimensions, although the method can in principle be applied in any dimension. The method relies on representing the solution of the initial-value problem for the parabolic equation via 


\section{L . EDWARDS}

its Green's function and suitable multiple reflections across the boundary of the domain. Though this process, sometimes known as the method of images[1], we extend the the solution to the whole space in such a way that our extension satisfies the prescribed boundary conditions. The resulting solution is periodic on the plane. As in $[2,3]$, our method relies on writing the solution $u(t, x)=\int G(t, x, y) f(y) d y$, where $f$ is the initial data and $G$ is the Green function of our problem. In the whole plane, the Green's function is more often referred to as the fundamental solution. The main issue in the variable coefficient case is to approximate $G$. A solution to this problem is proposed in $[2,3]$, where equations on domains without boundaries were considered, and hence no boundary conditions were needed. A natural question is whether the approximation in $[2,3]$ recovers the exact solution in the case of constant coefficients, for which the fundamental solution is known. Another natural question is how should the method be modified in the presence of boundaries. These two questions are addressed in this paper in the particular case of a rectangular domain and the approximation of solutions to the heat equation in two space dimensions. It should also be noted that the method is applicable in situations where we have coefficients that are constant at the boundary but may be non-constant within the domain of interest by combining the approximations discussed here with those in $[2,3]$ for the variable-coefficient case. Another general issue, is to estimate the integrals $\int G(t, x, y) f(y) d y$ numerically, and for this we use the simplest approach of a composite trapezoid rule for periodic functions, since there seems to be no additional advantage in considering some higher order rules. As a means for comparison against our method, we utilize an alternating direction implicit (ADI) method due to Peaceman and Rachford [4] to numerically solve the initial-value problem directly.

We study the initial-boundary-value problem for the two-dimensional heat equation on the square $0 \leq x, y \leq \pi$, given by

$$
\frac{\partial u}{\partial t}=\frac{\partial^{2} u}{\partial x^{2}}+\frac{\partial^{2} u}{\partial y^{2}} \quad, \quad 0 \leq x, y \leq \pi
$$

where we will consider the imposed Dirichlet boundary conditions

$$
u(t, x, 0)=u(t, x, \pi)=u(t, 0, y)=u(t, \pi, y)=0
$$

and an initial condition of the form

$$
u(0, x, y)=f(x, y):=\sum_{m=1,2 \ldots n=1,2 \ldots} \sum_{m n} \sin (n x) \sin (m y) .
$$

with suitable coefficients $A_{m n} \in \mathbb{R}$.

The existence of a closed form solution to this problem makes it especially useful in demonstrating the robustness of our numerical method since we can always compare with the exact solution. The exact solution given the boundary and initial conditions is well known to be

$$
u(t, x, y)=\sum_{m=1,2 \ldots} \sum_{n=1,2 \ldots} A_{m n} \sin (n x) \sin (m y) e^{-\left(n^{2}+m^{2}\right) t} .
$$

We utilize a combination of absolute and relative forms of the discretized $L^{2}$ and $L^{\infty}$ error norms in order to obtain a complete understanding of the strengths of our method over others. 
In the future, it would be interesting to apply the methods discussed in this paper to higher dimensional cases. Since the two dimensional calculations used in this paper are primarily useful as a simple platform for discussion, an application to higher dimensional problems would provide a more complete picture of the robustness of the method. It would also be interesting to consider the extension of the method to equations with non-constant coefficients, but this process is beyond the scope of this report.

We begin our discussion by defining the solution to the initial-boundary-value problem discussed above utilizing the Green's function. This is followed by a demonstration of the process for reflecting the calculation across the boundary of the square and extending the solution to the entire plane in an odd and periodic way. The extended solution can be written as convolution with the fundamental solution, also called the heat kernel. We then show how the fundamental solution in two dimensions can be equated to a product of one dimensional fundamental solutions.

The next section provides the details of how we move from the closed form solution just derived to a finite approximation that is more manageable in a numerical setting. Thus, while in our simple, model problem, the fundamental solution (or Green function) can be determined explicitly, we still find it useful to approximate it with a simpler function. We expect this to be quite useful for higher order problems. Methods for simplifying and increasing the efficiency of our method from a computational standpoint are discussed.

This is followed by a brief discussion of the Peaceman-Rachford ADI method against which we will compare the Green's function method. It is a relatively simple implicit predictor-corrector method for solving PDEs in multiple spacial dimensions which is unconditionally stable (in two dimensions) and is still used in some applications[4]. We also lay out the precise details of how the two methods are compared against one another in terms of speed (operation cost) and accuracy.

Finally, we provide a discussion of our results along with a comparison of error and operation count data, highlighting the strengths of our method.

\section{Fundamental Solution Approach}

We now show the derivation of the solution to the two-dimensional heat equation using the Green's function. The formulation will lead to the observation that the formula can be extended to arbitrarily many spacial dimensions by simply taking the product of one dimensional fundamental solutions.

The strategy will be to reflect this calculation across the boundary and extend it to the the whole plane in an odd and periodic fashion, thus obtaining the expression for the extended solution (3) as an infinite series of definite integrals. Importantly, the resulting series will be rapidly convergent, allowing for the truncation of the series to retain only a small finite number of its terms without a significant loss of accuracy in the approximation. The details of this truncation are discussed in Section 3. This approach is related to what is known as Ewald summation, a commonly used tool in electrostatics and computational chemistry for studying systems that are infinite and periodic. The fundamental idea behind Ewald summation is to break up a single divergent series into the sum of two series, one that is summable in the Fourier domain, and one that is summable with rapidly decaying terms in the real domain. This allows the energy of an infinite system to be approximated accurately and efficiently with truncated sums, which in turn leads to highly efficient numerical algorithms that take advantage of this rapid convergence [5]. 
The solution $u(t, x, y)$ of the initial-value for the heat equation in the whole plane is given by the convolution of the fundamental solution $G$ with the initial condition $f$ (see e.g. [6])

$$
u(t, x, y)=\int_{-\infty}^{\infty} \int_{-\infty}^{\infty} G(t, x, y, z, v) f(z, v) d z d v
$$

where the fundamental solution $G$ is given by:

$$
G(t, x, y, z, v)=\frac{1}{4 \pi t} \exp \left[\frac{-(x-z)^{2}-(y-v)^{2}}{4 t}\right] .
$$

We want to obtain from this explicit form of the solution in the entire plane a form of the solution of our initial-value/Dirichlet boundary value problem. To this end, we first observe that the indefinite integral (3) can be written as the infinite sum of definite integrals in the following way:

$$
\begin{gathered}
u(t, x, y)=\left(\cdots+\int_{0}^{\pi} \int_{0}^{\pi}(G \cdot f) d z d v+\int_{0}^{\pi} \int_{-\pi}^{0}(G \cdot f) d z d v+\cdots\right. \\
\left.\cdots+\int_{-\pi}^{0} \int_{0}^{\pi}(G \cdot f) d z d v+\int_{-\pi}^{0} \int_{-\pi}^{0}(G \cdot f) d z d v+\cdots\right)
\end{gathered}
$$

where we have written $(G \cdot f)$ for $G(t, x, y, z, v) f(z, v)$, for simplicity.

This integral can be rewritten as the double infinite summation:

$$
\begin{array}{r}
u(t, x, y)=\frac{1}{4 \pi t} \sum_{k, m=-\infty}^{\infty}\left[\int_{2 m \pi}^{(2 m+1) \pi} \int_{2 k \pi}^{(2 k+1) \pi}(G \cdot f) d z d v+\int_{2 m \pi}^{(2 m+1) \pi} \int_{(2 k-1) \pi}^{2 k \pi}(G \cdot f) d z d v\right. \\
\left.\cdots+\int_{(2 m-1) \pi}^{2 m \pi} \int_{2 k \pi}^{(2 k+1) \pi}(G \cdot f) d z d v+\int_{(2 m-1) \pi}^{2 m \pi} \int_{(2 k-1) \pi}^{2 k \pi}(G \cdot f) d z d v\right] .
\end{array}
$$

The convergence of this sum is justified by the convergence of the original integral in the whole plane, which in turns depends on having the right summability assumptions on the initial data $f$. Importantly, $f$ is typically very nice in applications (continuous and with compact support), so we will neglect providing the optimal assumptions on $f$ in what follows.

We utilize the homogeneous Dirichlet boundary conditions and extend $u$ to an everywhere defined periodic function (with period $2 \pi$ in each variable) by requiring that $u$ be odd with respect to reflections across the the walls of our domain. Accordingly, we first make the substitutions $z=$ $z+2 m \pi$ and $v=v+2 k \pi$ in our equation. Since our initial condition $f$ is $2 \pi$ periodic, we have:

$$
f(z+2 m \pi, v+2 k \pi)=f(z, v) .
$$

By making a change of variables in each integral, we can rewrite (6) as follows:

$$
\begin{array}{r}
u(t, x, y)=\frac{1}{4 \pi t} \sum_{k=-\infty}^{\infty} \sum_{m=-\infty}^{\infty}\left[\int_{0}^{\pi} \int_{0}^{\pi}\left(G_{m, k} \cdot f\right) d z d v+\int_{0}^{\pi} \int_{-\pi}^{0}\left(G_{m, k} \cdot f\right) d z d v+\cdots\right. \\
\left.\cdots+\int_{-\pi}^{0} \int_{0}^{\pi}\left(G_{m, k} \cdot f\right) d z d v+\int_{-\pi}^{0} \int_{-\pi}^{0}\left(G_{m, k} \cdot f\right) d z d v\right] .
\end{array}
$$


where the translated Green's function $G_{m, k}$ has the formula:

$$
G_{m, k}=\frac{1}{4 \pi t} \exp \left[\frac{-(x-z-2 m \pi)^{2}-(y-v-2 k \pi)^{2}}{4 t}\right] .
$$

Now, we make the substitutions $z=-z$ and $v=-v$ in the terms that corresponds to integration over the negative interval, using that the extension of $f$ is odd. We can then reverse the order of the bounds on the integrals, make the appropriate sign changes, and combine the terms under one double integral. The solution becomes

$$
u(t, x, y)=\frac{1}{4 \pi t} \sum_{k=-\infty}^{\infty} \sum_{m=-\infty}^{\infty}\left[\int_{0}^{\pi} \int_{0}^{\pi}\left(\left(e^{-A_{m}}-e^{-B_{m}}\right)\left(e^{-C_{k}}-e^{-D_{k}}\right) \cdot f(z, v) d z d v\right)\right]
$$

where $A_{m}$ and $B_{m}$ are defined as

$$
A_{m}=\frac{(x-z-2 m \pi)^{2}}{4 t}, \quad B_{m}=\frac{(x+z-2 m \pi)^{2}}{4 t}
$$

and $C_{k}$ and $D_{k}$ are defined as

$$
C_{k}=\frac{(y-v-2 m \pi)^{2}}{4 t}, \quad D_{k}=\frac{(y+v-2 m \pi)^{2}}{4 t} .
$$

Now, observe that if we define $H_{m}(t, x, z)$ and $H_{k}(t, y, v)$ in the following manner:

$$
H_{m}(t, x, z)=e^{-A_{m}}-e^{-B_{m}}, \quad H_{k}(t, y, v)=e^{-C_{k}}-e^{-D_{k}},
$$

then the solution can be written as

$$
u(t, x, y)=\frac{1}{4 \pi t} \sum_{k=-\infty}^{\infty} \sum_{m=-\infty}^{\infty}\left[\int_{0}^{\pi} \int_{0}^{\pi}\left(H_{m}(t, x, z) \cdot H_{k}(t, y, v) f(z, v)\right) d z d v\right],
$$

Let us denote $G^{(1)}(t, x, z):=\frac{1}{\sqrt{4 \pi t}} \sum_{m=-\infty}^{\infty} H_{m}(t, x, z)$ and

$$
G^{(2)}(t, x, y, z, v):=G^{(1)}(t, x, z) G^{(1)}(t, y, v)
$$

Then formula (10) becomes

$$
u(t, x, y)=\int_{0}^{\pi} \int_{0}^{\pi} G^{(2)}(t, x, y, z, v) f(z, v) d z d v
$$

So far, our calculations have been formal, but they can be justified by estimating the terms involved. These estimations will show that the corresponding series are absolutely and uniformly summable and, more importantly for applications, they will give a more effective way of approximating the Green's function $G^{(2)}$.

We provide the necessary estimations of the exponential terms (9) in the following proposition. 


\section{EDWARDS}

Proposition 2.1. Let $K:=e^{-\pi^{2} / 4 t}$. Then for the exponential terms in (8), we have the following uniform bounds. For $m, k \in \mathbb{Z}$ we have

$$
e^{-A_{m}} \leq K^{|m|}, \quad \text { and } \quad e^{-C_{k}} \leq K^{|k|} .
$$

For $m, k \in \mathbb{Z}$, with $m, k \neq 1$, we have

$$
e^{-B_{m}} \leq K^{|m|}, \quad \text { and } \quad e^{-D_{k}} \leq K^{|k|}
$$

Proof. We demonstrate the bounds for $e^{-A_{m}}$ and $e^{-B_{m}}$. The corresponding bounds for $e^{-C_{k}}$ and $e^{-D_{k}}$ follow from identical arguments. Note that for $m=0$ we have $e^{-A_{0}}=e^{-B_{0}}=K^{0}=1$. For all other $m \in \mathbb{Z}$, using the fact that $x, z \in[0, \pi]$, we have the following string of inequalities:

$$
\begin{aligned}
e^{-A_{m}}=\exp \left(-\frac{(-2 m \pi+x-z)^{2}}{4 t}\right) & \leq \exp \left(-\frac{(2 m \pi-\pi)^{2}}{4 t}\right) \\
& \leq \exp \left(-\frac{(m \pi)^{2}}{4 t}\right) \leq \exp \left(-\frac{\pi^{2}}{4 t}\right)^{|m|}=K^{|m|}
\end{aligned}
$$

To bound $e^{-B_{m}}$, we note that for $m=0$ and $m=1$, we have $e^{-B_{m}} \leq 1$. For all other $m \in \mathbb{Z}$, we can make the following estimates

$$
e^{-B_{m}}=\exp \left(-\frac{(-2 m \pi+x+z)^{2}}{4 t}\right) \leq \exp \left(-\frac{(m \pi)^{2}}{4 t}\right) \leq \exp \left(-\frac{\pi^{2}}{4 t}\right)^{|m|}=K^{|m|}
$$

This completes the proof.

We note that the factor $K$ in the above proposition depends on $t$ and is very small as $t \rightarrow 0$ : $\lim _{t \rightarrow 0} K=0$, but $K$ increases to 1 as $t$ increases to $\infty$.

Let us fix an integer $N \geq 0$ in the following discussion and let us define $\tilde{G}^{(1)}(t, x, z):=\frac{1}{\sqrt{4 \pi t}} \sum_{|m| \leq N} H_{m}(t, x, z)$ and

$$
\tilde{G}^{(2)}(t, x, y, z, v):=\tilde{G}^{(1)}(t, x, z) \tilde{G}^{(1)}(t, y, v) .
$$

Then we have the following estimates:

Theorem 2.2. Let $t>0$ and $K:=e^{-\pi^{2} / 4 t}$, as in Proposition 2.1, and $D:=(\sqrt{\pi t}(1-K))^{-1}$. Then

(i) $\left|G^{(1)}(t, x, z)\right| \leq 2 D$ and $\left|\tilde{G}^{(1)}(t, x, z)\right| \leq 2 D\left(1-K^{N+1}\right)$ for all $x, z$.

(ii) $\left|G^{(1)}(t, x, z)-\tilde{G}^{(1)}(t, x, z)\right| \leq D K^{N+1}$.

(iii) $\left|G^{(2)}(t, x, y, z, v)-\tilde{G}^{(2)}(t, x, z)\right| \leq 2 D^{2} K^{N+1}$. 
Proof. (i) From the definition of $G^{(1)}$ and $\tilde{G}^{(1)}$, the bounds in Proposition 2.1, and the expression for the sum of a convergent geometric series, we have

$$
\left|G^{(1)}(t, x, z)\right| \leq \frac{2}{\sqrt{4 \pi t}} \sum_{m=-\infty}^{\infty}\left|H_{m}(t, x, z)\right| \leq \frac{4}{\sqrt{4 \pi t}} \sum_{m=0}^{\infty} K^{m}=2 D
$$

and similarly,

$$
\left|\tilde{G}^{(1)}(t, x, z)\right| \leq \frac{2}{\sqrt{4 \pi t}} \sum_{|m| \leq N}\left|H_{m}(t, x, z)\right| \leq \frac{4}{\sqrt{4 \pi t}} \sum_{m=0}^{N} K^{m}=2 D\left(1-K^{N+1}\right) .
$$

(ii) This follows immediately from (i).

(iii) This last estimate is obtained by writing

$$
\begin{aligned}
G^{(2)}(t, x, y, z, v)-\tilde{G}^{(2)}(t, x, y, z, v)=\left(G^{(1)}(t, x, z)\right. & \left.-\tilde{G}^{(1)}(t, x, z)\right) G^{(1)}(t, y, v) \\
& +\tilde{G}^{(1)}(t, x, z)\left(G^{(1)}(t, y, v)-\tilde{G}^{(1)}(t, y, v)\right),
\end{aligned}
$$

and then using the results from (i) and (ii). This completes the proof.

The following proposition serves to characterize the behavior of our approximation.

Proposition 2.3. We have $\lim _{t \rightarrow 0} 2 D^{2} K^{N+1} / t^{k}=0$ for all $k>0$.

Proof. Observe

$$
\lim _{t \rightarrow 0} \frac{2 D^{2} K^{N+1}}{t^{k}}=\lim _{t \rightarrow 0} \frac{2 K^{N+1}}{\pi t(1-K)^{2} t^{k}} \leq \lim _{t \rightarrow 0} \frac{K^{N+1}}{K^{2} t^{k+1}}=\lim _{t \rightarrow 0} \frac{K^{N-1}}{t^{k+1}}=0 .
$$

The last equality is clear from the dominance of the exponential term $K$ which goes to 0 faster than any polynomial in $t$ as $t \rightarrow 0$.

We thus obtain a very good approximation as $t \rightarrow 0$, i.e. for small time. However, this approximation is not very good for very large $t$. We can improve it however for medium $t$ by increasing $N$. This is why in our numerical tests we take $N=1$ instead of $N=0$.

Now, for some applications it may be of interest to write our solution as the product of two terms, independent of one another with respect to the spatial variables $x$ and $y$. This can be done if the initial data can be expressed as a separable product. We provide this result as a proposition. We stress that our method works in general for any sufficiently regular $f$.

Proposition 2.4. The fundamental solution $G^{(2)}$ to the heat equation in two dimensions can be expressed as the product of one dimensional solutions as in Equation (11). Consequently, the solution $u(t, x) \cdot u(t, y)$ for a continuous initial value $f(z, v)=f_{1}(z) f_{2}(v)$ can be written as 


$$
u(t, x, y)=\left(\int_{0}^{\pi} G^{(1)}(t, x, z) f_{1}(z) d z\right)\left(\int_{0}^{\pi} G^{(1)}(t, y, v) f_{2}(v) d v\right) .
$$

Proof. In order to assert this equality, it is enough to show uniform convergence of the series

$$
\sum_{k=-\infty}^{\infty} \sum_{m=-\infty}^{\infty}\left[\left(\left(e^{-A_{m}}-e^{-B_{m}}\right)\left(e^{-C_{k}}-e^{-D_{k}}\right) \cdot f(z, v) d z d v\right)\right],
$$

over the region $[0, \pi] \times[0, \pi]$. Using the bounds given in Proposition 2.1 and the fact that convergence is preserved under addition and multiplication of absolutely convergent series, the uniform convergence of (17) is immediate from the Weierstrass M test.

This proof lays the foundation for an important result which we will offer as a proposition, but will not prove here. Indeed, it can be shown that the previous result can be extended to arbitrarily many spacial dimensions.

Proposition 2.5. The Green function for the heat equation with zero Dirichlet boundary conditions on the Cartesian product of $n$ intervals is the product of the corresponding Green functions for each interval. Moreover, if the initial data has a product structure $f\left(x_{1}, x_{2}, \ldots, x_{n}\right)=$ $f_{1}\left(x_{1}\right) f_{2}\left(x_{2}\right) \ldots f_{n}\left(x_{n}\right)$, then the solution of the $n$-dimensional problem is the product of $n$ onedimensional solutions $u_{i}\left(t, x_{i}\right)$ in $x_{i}$, that is,

$$
u\left(t, x_{1}, x_{2}, \ldots, x_{n}\right)=u_{1}\left(t, x_{1}\right) u_{2}\left(t, x_{2}\right) \ldots u_{n}\left(t, x_{n}\right) .
$$

This result could be of importance in practical applications with separable initial data, as it is often necessary to consider in higher dimensional problems.

\section{Numerical Method}

While the solution (12) just developed is exact, it is not feasible or necessary to calculate the infinite sums numerically. The goal is to utilize this solution to numerically approximate the exact solution to the heat equation using a far smaller operation count than other known methods. The operation count for a calculation is found by tracking the number of operations that a program must execute in order to produce an estimate within a given threshold of accuracy of the exact solution. It should be noted that there is necessarily some error due to computer rounding when calculating the exact solution, but it is negligible and will not be a relevant factor in our comparisons.

Several observations can be made about our solution which significantly reduce the operation count while preserving the accuracy of the approximation.

Recall that the solution (12) is found by taking the infinite sum of the integral of a product of terms similar in form to the expression

$$
\exp \left[\frac{-(x+z-2 m \pi)^{2}}{4 t}\right]
$$


Based on the results given at the end of Section 2, we know these terms exhibit rapid decay everywhere on the domain $[0, \pi] \times[0, \pi]$ as $|m|$ grows large. This can also be easily observed informally from (18) by fixing some small $t>0$ and $x, z$ in the domain and evaluating the exponential for increasing values of $m$. This rapid decay is of critical importance for the efficiency of our numerical method. It turns out that all but a small finite number of terms in the series are negligible, thus the infinite sums can be truncated dramatically while still providing very accurate approximations. In particular, the calculations in this report were done by truncating the sums so that they only include terms corresponding to $m, k \in\{0, \pm 1\}$.

Thus the approximation to (12) used to obtain all of the data in this report is given by

$$
u(t, x, y) \approx \frac{1}{4 \pi t} \sum_{m, k=0, \pm 1}\left[\int_{0}^{\pi} \int_{0}^{\pi}\left(H_{m}(t, x, z) H_{k}(t, y, v) f(z, v)\right) d z d v\right]
$$

This formula allows for a significant reduction in operation count without significantly compromising the accuracy of the approximation. In fact, using the product structure, we see that we need to compute only 12 exponentials.

To approximate the integrals in the approximate solution, we use a standard midpoint Riemann sum, which turns out to be sufficient despite its relative simplicity (second order), even with a surprisingly low number of partitions. For periodic functions, the composite midpoint rule will coincide with the composite trapezoid rule, and it seems that higher order composite methods actually do not contribute to the precision of the integration.

In order to balance the accuracy with the operation count of our method, the fineness of the mesh used to approximate the integrals in the approximation (19) can be increased or decreased. The number of terms of the series can also be increased based on the desired accuracy of the approximation. Thus, we can easily alter the method to better the approximation while maintaining the minimum operation count that meets our needs.

\section{Comparison Methods}

In order to obtain a comparison of the accuracy vs. speed of our solution with other known methods in the two-dimensional case, we use a Peaceman-Rachford variant of the ADI method to solve the PDE directly. Comparisons with explicit and implicit Euler schemes in the one-dimensional case have been made by W. Cheng et al. [2]. The ADI method is best known for its use in solving heat conduction problems. It is a finite-difference based scheme which splits the solution into two parts. First, an implicit treatment of the first space variable is utilized while the other is treated explicitly. These roles are then reversed in the next step. The method can be shown to be unconditionally stable with respect to the time and space discretizations for two dimensional problems, and is second order in time and space [4].

The strength of the ADI method is partially due to the fact that it reduces to solving a symmetric tridiagonal system, unlike the well known implicit Crank-Nicolson method from which it

is derived. Due to this nice structure, the number of operations per time step needed to find a 
solution on an $N \times M$ rectangular grid is only $\mathcal{O}(N M)$. However, $\mathcal{O}\left((N M)^{2}\right)$ time steps are usually required. The Greens function method requires a total of $\mathcal{O}\left((N M)^{2}\right)$ operations to obtain a solution on the same rectangular grid. This assumes that the discretization of the integrals in (19) is $\mathcal{O}(N M)$, which is typically an overestimate. It is important to emphasize that there is no time stepping needed in the Greens function method, although a small number of time steps may be taken when using the approximate Greens function in order to keep errors small. It should be noted that although (19) does not converge to the exact solution as $N$ and $M$ go to infinity if the number of time steps is fixed, we do obtain convergence by allowing the number of time steps to go to infinity. The number of time steps needed to achieve a certain error is nevertheless much smaller than for the ADI method.

The two major steps of the ADI algorithm applied to the two-dimensional heat equation can be expressed concisely as follows:

$$
\begin{gathered}
\frac{u_{i j}^{n+\frac{1}{2}}-u_{i j}^{n}}{\Delta t / 2}=\left[\delta_{x}^{2} u_{i j}^{n+\frac{1}{2}}+\delta_{y}^{2} u_{i j}^{n}\right] \\
\frac{u_{i j}^{n+1}-u_{i j}^{n+\frac{1}{2}}}{\Delta t / 2}=\left[\delta_{x}^{2} u_{i j}^{n+1}+\delta_{y}^{2} u_{i j}^{n+\frac{1}{2}}\right],
\end{gathered}
$$

where $\delta_{x}^{2}$ and $\delta_{y}^{2}$ represent second order central difference operators in $x$ and $y$ respectively, e.g.

$$
u_{x x} \rightarrow \frac{\delta_{x}^{2} u_{i j}^{n}}{h^{2}} \equiv \frac{u_{i+1, j}^{n}-2 u_{i, j}^{n}+u_{i-1, j}^{n}}{h^{2}} .
$$

The subscripts $i, j$ in these formulas correspond to the spatial discretization and the superscript $n$ corresponds to the time step. Note that the first step (20) is implicit in $x$ and explicit in $y$, whereas the second (21) is implicit in $y$ and explicit in $x$. In fact, the implicit and explicit schemes are simply backward and forward Euler respectively. The algorithm proceeds by conducting alternating sweeps, first in the $x$ direction at half time steps $n \frac{\Delta t}{2}$, then in the $y$ direction at times $n \Delta t$.

In order to compare the ADI method against the Green's function method, we first obtain the exact closed form solution as well as both approximations to the solution of the heat equation on $[0, \pi] \times[0, \pi]$ given some initial condition. We then determine the (discretized) $L^{2}$ and $L^{\infty}$ error for each method at some specified time. Note that although the spatial discretization for the ADI method was not necessarily fixed between trials, the error calculations were only made on a $50 \times 50$ grid over the square. For consistency, the same grid was then used to calculate the error in the approximation found using the Greens function method.

Both absolute and relative forms of these errors will be used in order to present a clearer picture of the results. Our first two comparisons are meant to highlight the relatively small operation count required by the Green's function method as compared to the ADI method in obtaining a reasonably accurate solution. We consider two different initial conditions, the first consisting of a single low frequency mode in each of $x$ and $y$, and the second consisting of five modes of widely varying frequencies. We then focus on the high order accuracy that can be obtained using the Green's function method while still maintaining a manageable operation count. 
Finally, It is important to note that although the algorithm for the Green's function method can be made faster by utilizing the one-dimensional nature of the solutions given certain initial conditions, we do not expect to have these conditions in general and thus we conduct the comparisons without taking advantage of this computational shortcut. Thus, the comparisons made here are valid for a much broader class of initial conditions, in particular, those that cannot be separated.

\section{Results and Conclusions}

The superiority of our method over the ADI method in terms of speed and accuracy is demonstrated in several different ways. Tables 1 and 2 are meant to demonstrate the comparatively low operation cost or speed of our method when obtaining a reasonably accurate solution. To obtain the results in these tables, each method was used to obtain approximations with relative $L^{\infty}$ error of $\mathrm{O}\left(10^{-4}\right)$ on the domain while maintaining the lowest possible operation count. In the approximations obtained using the Greens function method, only the number of partitions used in approximating the integrals in (19) were changed in order to increase accuracy. For the ADI method, both the time and spatial discretizations were systematically and individually incremented by \pm 10 in order to experimentally obtain the lowest order operation count for a desired accuracy. Note that the data in Table 1 correspond to a single mode initial condition and the data in Table 2 correspond to a more peaked initial condition with five modes. All of the errors given in Tables 1 and 2 are relative errors, and the operation counts are approximate.

Table 1 provides a comparison of the two methods in solving the heat equation with the initial condition $f(x, y)=\sin (3 x) \sin (3 y)$. Both methods require $\mathrm{O}\left(10^{5}\right)$ operations to arrive at a solution within the required error tolerance for early time $t=0.05$, but this trend does not persist. The Green's function method requires roughly one order of magnitude fewer operations at intermediate times $t=0.10$ and $t=0.50$, and three orders of magnitude fewer at a 'late' time $t=1.00$. Also, note that the two methods differ dramatically in the number of operations required as time increases. To arrive at a solution at any given time $t_{i}$, the ADI method must compute the solution at a certain number of earlier times $t_{1}, \ldots, t_{i-1}$ depending on the time step chosen. This means that obtaining a solution at a later time typically requires many more operations than an earlier time. This is not the case with the Green's function method which does not have any dependence on earlier data. Thus the operation count for the ADI method increases with time whereas the operation count decreases for the Green's function method as the solution becomes less peaked.

\begin{tabular}{|l|c|c|c|c|c|c|}
\hline Time & \multicolumn{2}{|c|}{ Relative $L^{2}$ Error } & \multicolumn{2}{c|}{ Relative $L^{\infty}$ Error } & \multicolumn{2}{c|}{ Operation Count } \\
\hline & Green's & ADI & Green's & ADI & Green's & ADI \\
\hline $\mathbf{0 . 0 5}$ & $4.90 \mathrm{e}-3$ & $2.53 \mathrm{e}-2$ & $2.46 \mathrm{e}-4$ & $9.92 \mathrm{e}-4$ & $1.22 \mathrm{e} 5$ & $1.20 \mathrm{e} 5$ \\
\hline $\mathbf{0 . 1 0}$ & $4.82 \mathrm{e}-3$ & $2.20 \mathrm{e}-2$ & $2.59 \mathrm{e}-4$ & $9.86 \mathrm{e}-4$ & $9.00 \mathrm{e} 4$ & $4.35 \mathrm{e} 6$ \\
\hline $\mathbf{0 . 5 0}$ & $4.66 \mathrm{e}-5$ & $1.11 \mathrm{e}-2$ & $1.10 \mathrm{e}-5$ & $9.99 \mathrm{e}-4$ & $4.00 \mathrm{e} 4$ & $6.38 \mathrm{e} 6$ \\
\hline $\mathbf{1 . 0 0}$ & $4.50 \mathrm{e}-3$ & $9.53 \mathrm{e}-3$ & $3.25 \mathrm{e}-4$ & $9.85 \mathrm{e}-4$ & $4.00 \mathrm{e} 4$ & $1.69 \mathrm{e} 7$ \\
\hline
\end{tabular}

TABLE 1. Relative $L^{2}$ and $L^{\infty}$ errors and operation count required for each method in obtaining an approximation with $\mathrm{O}\left(10^{-4}\right)$ relative $L^{\infty}$ error at various times. Initial condition: $f(x, y)=\sin (3 x) \sin (3 y)$.

Table 2 contains data corresponding to similar calculations, but with the initial condition 


$$
f(x, y)=\sin (3 x) \sin (3 y)-\sin (2 x) \sin (4 y)-\sin (5 x) \sin (2 y)-\sin (7 x) \sin (5 y)+2 \sin (6 x) \sin (10 y),
$$

with modes chosen more or less arbitrarily as a representative for more complicated or more peaked initial data. (Note that an earlier time, $t=.01$, is included to reflect the smaller time scales inherent in the problem for this initial condition.) As expected, both methods require more operations to reach the same $L^{\infty}$ error tolerance than with the single mode initial condition. However, the increase is not as dramatic for the Green's function method as it is for the ADI method which requires roughly an order of magnitude more operations than with the previous initial condition. Again, we see the two methods exhibiting opposite trends in terms of operation count with respect to the time at which the solution is being approximated.

\begin{tabular}{|c|c|c|c|c|c|c|}
\hline Time & \multicolumn{2}{|c|}{ Relative $L^{2}$ Error } & \multicolumn{2}{c|}{ Relative $L^{\infty}$ Error } & \multicolumn{2}{c|}{ Operation Count } \\
\hline & Green's & ADI & Green's & ADI & Green's & ADI \\
\hline $\mathbf{0 . 0 1}$ & $1.34 \mathrm{e}-2$ & $4.90 \mathrm{e}-3$ & $5.98 \mathrm{e}-4$ & $9.94 \mathrm{e}-4$ & $9.02 \mathrm{e} 5$ & $3.92 \mathrm{e} 6$ \\
\hline $\mathbf{0 . 0 5}$ & $1.54 \mathrm{e}-2$ & $6.81 \mathrm{e}-3$ & $6.13 \mathrm{e}-4$ & $9.99 \mathrm{e}-4$ & $3.02 \mathrm{e} 5$ & $1.56 \mathrm{e} 6$ \\
\hline $\mathbf{0 . 1 0}$ & $1.54 \mathrm{e}-2$ & $7.73 \mathrm{e}-3$ & $6.12 \mathrm{e}-4$ & $9.90 \mathrm{e}-4$ & $2.02 \mathrm{e} 5$ & $3.17 \mathrm{e} 6$ \\
\hline $\mathbf{0 . 5 0}$ & $2.65 \mathrm{e}-3$ & $5.77 \mathrm{e}-3$ & $1.04 \mathrm{e}-4$ & $9.98 \mathrm{e}-4$ & $9.00 \mathrm{e} 4$ & $3.57 \mathrm{e} 7$ \\
\hline
\end{tabular}

TABLE 2. Relative $L^{2}$ and $L^{\infty}$ errors and operation count required for each method in obtaining an approximation with $\mathrm{O}\left(10^{-4}\right)$ relative $L^{\infty}$ error at various times. Initial condition: $f(x, y)=\sin (3 x) \sin (3 y)-\sin (2 x) \sin (4 y)-\sin (5 x) \sin (2 y)-$ $\sin (7 x) \sin (5 y)+2 \sin (6 x) \sin (10 y)$.

Table 3 contains only data for the Green's function method and is meant to illustrate the accuracy that can be obtained with the method without sacrificing speed. These data were obtained by first experimentally determining the maximal order of accuracy attainable in solving the heat equation with initial condition (23) using the approximation (19), and then computing approximations which attain that order of accuracy while maintaining the lowest possible operation count. Observe that the operation cost for these approximations is only roughly double that of the approximations from Table 2. Note that the comparatively large relative error at time $t=0.50$ is due to the fact that the solution decays to near 0 everywhere on the domain by this time.

In theory, the ADI method can be used to obtain equally accurate approximations, albeit at a much greater operation cost. However, the effect of compounding error becomes overwhelming as the fineness of the spacial and temporal discretization in increased, making it difficult to obtain highly accurate approximations.

Finally, Figure 1 provides a visualization of the convergence of the Green's function method with respect to operation count. The plot depicts the relative $L^{2}$ and $L^{\infty}$ error corresponding to approximate solutions to the heat equation with initial condition (23) at time $t=0.10$. As the the operation count increases from $10^{5}$, the approximation converges to the exact solution exponentially.

One of the great advantages of our method, and an explanation for the huge advantage in operation cost when compared to the Peaceman-Rachford ADI and other methods, is its lack of dependence on earlier data. We can arrive at a solution at any 'large' time at no greater cost than a smaller time solution would require. This is not the case for a recursive method which requires a dramatic 
GREEN'S FUNCTION METHOD

\begin{tabular}{|c|c|c|c|c|c|}
\hline Time & \multicolumn{2}{|c|}{ Absolute Error } & \multicolumn{2}{c|}{ Relative Error } & Operation Count \\
\hline & $L^{2}$ & $L^{\infty}$ & $L^{2}$ & $L^{\infty}$ & \\
\hline $\mathbf{0 . 0 1}$ & $4.38 \mathrm{e}-9$ & $1.05 \mathrm{e}-9$ & $4.15 \mathrm{e}-9$ & $9.93 \mathrm{e}-10$ & $2.02 \mathrm{e} 6$ \\
\hline $\mathbf{0 . 0 5}$ & $1.22 \mathrm{e}-9$ & $2.50 \mathrm{e}-10$ & $2.99 \mathrm{e}-9$ & $6.15 \mathrm{e}-10$ & $6.40 \mathrm{e} 5$ \\
\hline $\mathbf{0 . 1 0}$ & $4.37 \mathrm{e}-10$ & $9.14 \mathrm{e}-11$ & $2.94 \mathrm{e}-9$ & $6.15 \mathrm{e}-10$ & $4.22 \mathrm{e} 5$ \\
\hline $\mathbf{0 . 5 0}$ & $4.97 \mathrm{e}-10$ & $1.00 \mathrm{e}-10$ & $6.24 \mathrm{e}-6$ & $1.25 \mathrm{e}-6$ & $1.60 \mathrm{e} 5$ \\
\hline
\end{tabular}

TABLE 3. Absolute and relative $L^{2}$ and $L^{\infty}$ errors corresponding to highest accuracy attainable with Green's function method at each time for multi-modal initial condition (20).

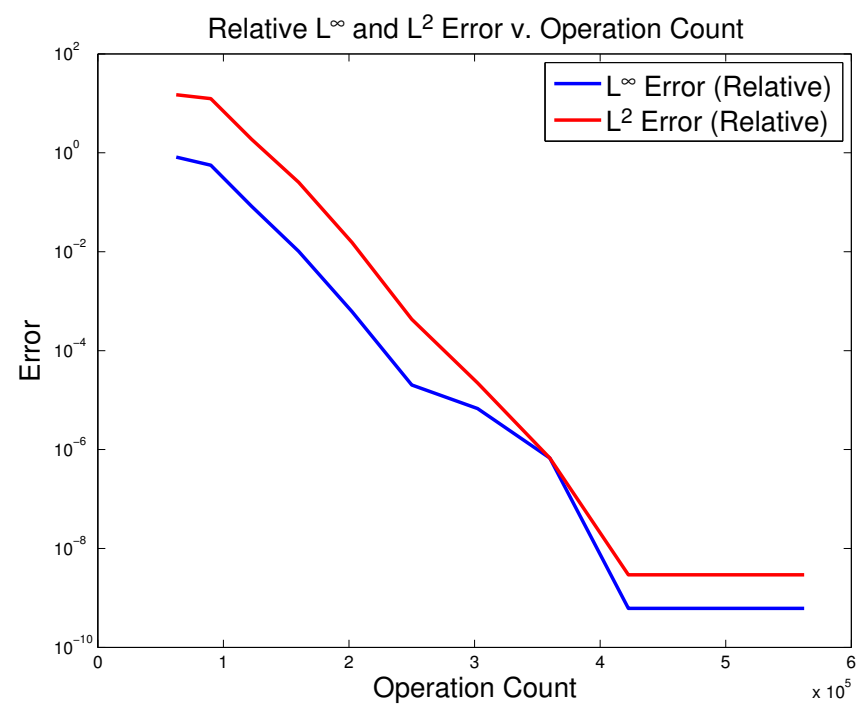

Figure 1. Relative error versus operation count for the Green's Function Method with initial condition $f(x)=\sin (4 x) \sin (4 y), t=0.1$.

increase in the number of operations with an increase in time just to maintain an accuracy consistent with earlier time approximations. This also means that with the Green's function method, we can approximate the solution at arbitrary times rather than a set of times dictated by the size of some time step $\Delta t$. Furthermore, the method, which is straightforward to implement even in high dimensions, affords us great control over operation count, making the minimization of calculation speed for a desired level of accuracy quite simple.

\section{REFERENCES}

[1] Tikhonov, A. N.; Samarskii, A. A. (1963). Equations of Mathematical Physics. New York: Dover Publications.

[2] W. Cheng, N. Costanzino, J. Liechty, A. Mazzucato, V. Nistor Closed-form asymptotics and numerical approximations of $1 D$ parabolic equations with applications to option pricing. SIAM J. Financial Math. 2 (2011), no. 1, 901-934. 


\section{EDWARDS}

[3] R. Constantinescu, N. Costanzino, A. Mazzucato, V. Nistor Approximate solutions to second order parabolic equations. I: analytic estimates. J. Math. Phys. 51 (2010), no. 10, 103502, 26 pp.

[4] W. H. Hundsdorfer and J. G. Verwer. Stability and Convergence of the Peaceman-Rachford Method for InitialBoundary Value Problems. J. Mathematics of Computation, Volume 53, Number 187. Pages 81-101. (1989).

[5] D. Lindbo and A.K. Tornberg, Fast and spectrally accurate Ewald summation for 2- periodic electrostatic systems. J. Chem. Phys., 136:164111, 2012.

[6] Hazewinkel, Michiel , ed. (2001), "Green function", Encyclopedia of Mathematics, Springer, ISBN 978-1-55608$010-4$ 